\title{
Mejoras en el factor de potencia mediante el reemplazo de tubos fluorescentes por tubos equivalentes LED
}

\section{Improvements in power factor by replacing fluorescent tubes with equivalent LED tubes}

\author{
José Luis Frund ${ }^{1}$, Agustin Chort ${ }^{2}$, Mónica $\mathrm{Krenz}^{3}$, Arturo Manuel Cassano ${ }^{4}$, Fabio Vincitorio ${ }^{5}$ \\ ${ }^{1}$ Grupo de Investigación de Electrónica de Potencia en Iluminación, Facultad Regional Paraná, Universidad Tecnológica \\ Nacional, Argentina. Email: pepefrund@gmail.com \\ ${ }^{2}$ Grupo de Investigación de Electrónica de Potencia en Iluminación, Facultad Regional Paraná, Universidad Tecnológica \\ Nacional, Argentina. Email: chort.agustin@gmail.com \\ ${ }^{3}$ Grupo de Investigación de Electrónica de Potencia en Iluminación, Facultad Regional Paraná, Universidad Tecnológica \\ Nacional, Argentina. Email: monicakrenz@ @otmail.com \\ ${ }^{4}$ Grupo de Investigación de Electrónica de Potencia en Iluminación, Facultad Regional Paraná, Universidad Tecnológica \\ Nacional, Argentina. Email: arturo.cassano@gmail.com \\ ${ }^{5}$ Grupo de Investigación de Electrónica de Potencia en Iluminación, Facultad Regional Paraná, Universidad Tecnológica \\ Nacional, Argentina. Email: fisicaelectronica@frp.utn.edu.ar
}

Recibido: octubre 19, 2017. Aceptado: diciembre 04, 2017. Versión final: febrero 20, 2018.

\begin{abstract}
Resumen
El reciente reemplazo de la iluminación del túnel sub fluvial Uranga - Silvestre Begnis, que une las ciudades de Paraná y Santa Fe en la República Argentina, ha planteado una serie de interrogantes técnicos respecto del reemplazo por nuevas tecnologías de iluminación. En esta obra, 4.200 tubos fluorescentes T8 de $36 \mathrm{~W}$ fueron reemplazados portubos de nueva Tecnología LED $14 \mathrm{~W}$. El reemplazo se fundamentó en la necesidad de reducir el consumo energético y lograr una reducción de los costos operativos. Entre las principales hipótesis antes de la obra, se evaluó un incremento de la distorsión armónica total, a partir de conectarcargas no lineales. Entre las especificaciones del pliego de obra se indicaron límites máximos de THD tolerables. Sin embargo, no se tuvo en cuenta la THD original propia del uso de balastos electromagnéticos con tubos fluorescentes. En este trabajo se muestran las mejoras detectadas en los parámetros de calidad eléctrica, a partir de la utilización de equipos LED con características adecuadas. Los resultados muestran una notable mejora del factor de potencia, con valores cercanos a 0,9 y una reducción del contenido armónico a niveles tan bajos como $18 \%$. Como efecto negativo se detectó la presencia de modulación de HF en la señal de corriente en el análisis individual de cada equipo. Sin embargo, en la instalación masiva es posible observar una notable reducción de la señal de pulsado propia de la configuración del driver.
\end{abstract}

Palabras clave: iluminación; factor de potencia; factor de desplazamiento; alta frecuencia; LED.

\begin{abstract}
The recent replacement of the illumination of the sub fluvial tunnel Uranga - Silvestre Begnis, that joins the cities of Paraná and Santa Fe in the Argentinean Republic, has raised a number of technical questions regarding the replacement for new lighting technologies. In this work, 4200 fluorescent tubes T8 of 36w were replaced by new technology LED tubes of $14 \mathrm{w}$. The replacement was based on the need to reduce energy consumption and to achieve a reduction in operating costs. Among the main hypothesis before the work, an increase in the total harmonic distortion from


connecting nonlinear loads was evaluated. Among the specifications of the statement work, maximum permissible THD limits were indicated. However, the original THD of the use of electromagnetic ballasts with fluorescent tubes was not take into consideration. This work shows the improvements detected in the parameters of electrical quality from the use of LED equipment with suitable characteristics. The results show a remarkable improvement of power factor with values close to 0.9 and a reduction of the harmonics content to levels as low as $18 \%$. As a negative effect, the presence of HF modulation was detected in the current signal in the individual analysis of each equipment. Nevertheless, in the mass installation process, it is possible to observe a noticeable reduction of the pulse signal characteristic of the driver configuration.

Keywor ds: lighting; power factor; displacement factor; high frequencies; LED.

(a)

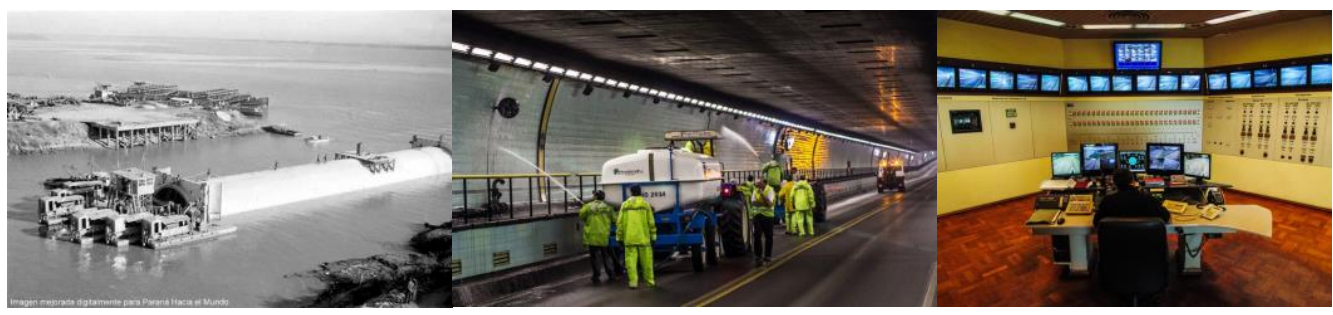

Figura 1. Fotos del complejo túnel sub fluvial Uranga - Silvestre Begnis. (a) construcción, (b) interior y (c) sala de control. Fuentes: (a) www.tunelsubfluvial.gov.ar, (b) www.welcomeargentina.com; (c) www.santafeturismo.gov.ar

\section{Introducción}

Las ciudades de Paraná y Santa Fe en la República Argentina se encuentran unidas por uno de los primeros túneles sub fluviales construidos en el mundo. Su construcción comenzó en el año 1.962 y la obra fue inaugurada en 1.969. Esto representó todo un logro de la tecnología disponible en aquellos días. Hoy representa una obra emblemática, no solo por lo que significa desde el punto de vista tecnológico sino porque permitió abandonar definitivamente el aislamiento de la provincia de Entre Ríos. Aislamiento impuesto por el río Paraná, uno de los 6 ríos de más caudal en el mundo. La provincia de Entre Ríos conforma junto con la de Corrientes y Misiones, una de las dos mesopotamias que existen en nuestro planeta. Desde el diseño del túnel, se estableció que no solo representaría una obra civil de magnitud extraordinaria para la época si no que la obra eléctrica y electrónica también lo serían. Sistemas de ventilación, iluminación, comunicaciones y circuito cerrado de televisión integran el conjunto de mayor desarrollo tecnológico, tanto en su construcción como en la actualidad.

En este contexto y habiendo pasado más de 45 años desde su construcción, la tecnología aplicada en el mantenimiento y en el funcionamiento del enlace vial sigue en permanente evolución. En este trabajo se documentan las mejoras registradas en el reemplazo del sistema de iluminación interior de la calzada. En agosto del 2016 se reemplazaron íntegramente los equipos auxiliares y los tubos fluorescentes de 4.200 luminarias. Para ello, se utilizaron tubos de nueva tecnología LED (tubos construidos con diodos emisores de luz), los que fueron seleccionados luego de rigurosos ensayos, superados solo por 5 fabricantes. Los tubos fueron instalados en las mismas luminarias alterando el cableado original para compatibilizarlo con los nuevos dispositivos. Sin embargo, permanecieron sin modificaciones los tendidos eléctricos de alimentación desde el edificio de mando hasta el interior del túnel. Así, los 4200 equipos de iluminación se encuentran conectados en dos circuitos trifásicos independientes, y se mantienen los sistemas de medición y de protección originales.

Por otra parte, esta instalación constituye un campo de pruebas ideal para este tipo de tecnología en iluminación. 4.200 equipos conectados 24 horas al día en circuitos eléctricos controlados, con condiciones ambientales favorables, funcionan bajo la supervisión de personal técnico altamente calificado.

\subsection{Tubos LED usados}

Para el reemplazo de los tubos fluorescentes originales un consultor privado seleccionó los tubos LED Philips MAS LEDtube $1200 \mathrm{~mm}$ SO 14,5W 865 T8 ROT, cuyas 
especificaciones se muestran en la tabla 1. En la hoja de datos puede verse que el fabricante declara un factor de potencia de 0,9 . Valor totalmente aceptable para cualquier norma internacional relacionada con el consumo de energía eléctrica en dispositivos de iluminación de baja potencia. Sin embargo, no declara valores relacionados con el factor de desplazamiento o valores que indiquen contenido de altas frecuencias moduladas en la corriente de línea.

Tabla 1. Detalles de los parámetros eléctricos principales del tubo Philips MAS LEDtube 1.200 mm SO 14,5W 865 T8 ROT

\begin{tabular}{|l|l|}
\hline \multicolumn{2}{|c|}{ Operating and Electrical } \\
\hline Input Frequency & 50 to $60 \mathrm{~Hz}$ \\
\hline Power (Rated) (Nom) & $14.5 \mathrm{~W}$ \\
\hline Starting Time (Nom) & $0,5 \mathrm{~s}$ \\
\hline Warm Up Time To 60\% Ligth (Nom) & inst ant full ligth \\
\hline Power Factor (Nom) & 0,9 \\
\hline Voltage (Nom) & $220-240 \mathrm{~V}$ \\
\hline
\end{tabular}

Fuente: Philips.

\subsection{Factor de potencia, factor de desplazamiento $y$ modulación de alta frecuencia}

Cuando se analizan cargas de iluminación que utilizan circuitos electrónicos auxiliares debe siempre considerarse que los factores de caracterización, simplificados en la ingeniería eléctrica de cargas lineales, presentan componentes no lineales en el consumo eléctrico. Así, el factor de potencia debe ser considerado como un factor mucho más amplio que el factor de desplazamiento o coseno phi [1].

$F P=\frac{P a c t}{P a p}=\frac{V_{e f} I_{e f 1}}{V_{e f} I_{e f}} \cos (\varphi)$

Según la ecuación 1, el factor de potencia queda definido por dos factores independientes: el factor de desplazamiento y el factor armónico que dependen de la magnitud de la corriente fundamental. Así, si la señal de corriente es armónicamente pura, el primer miembro del segundo término es idénticamente igual a 1 . Debido a que la corriente es no lineal, entonces la magnitud de la corriente fundamental será menor al de la corriente eficaz total y, por lo tanto, existirán componentes armónicas en la corriente consumida [2]. En la figura 2 se muestra un ejemplo de forma de onda de corriente no lineal.
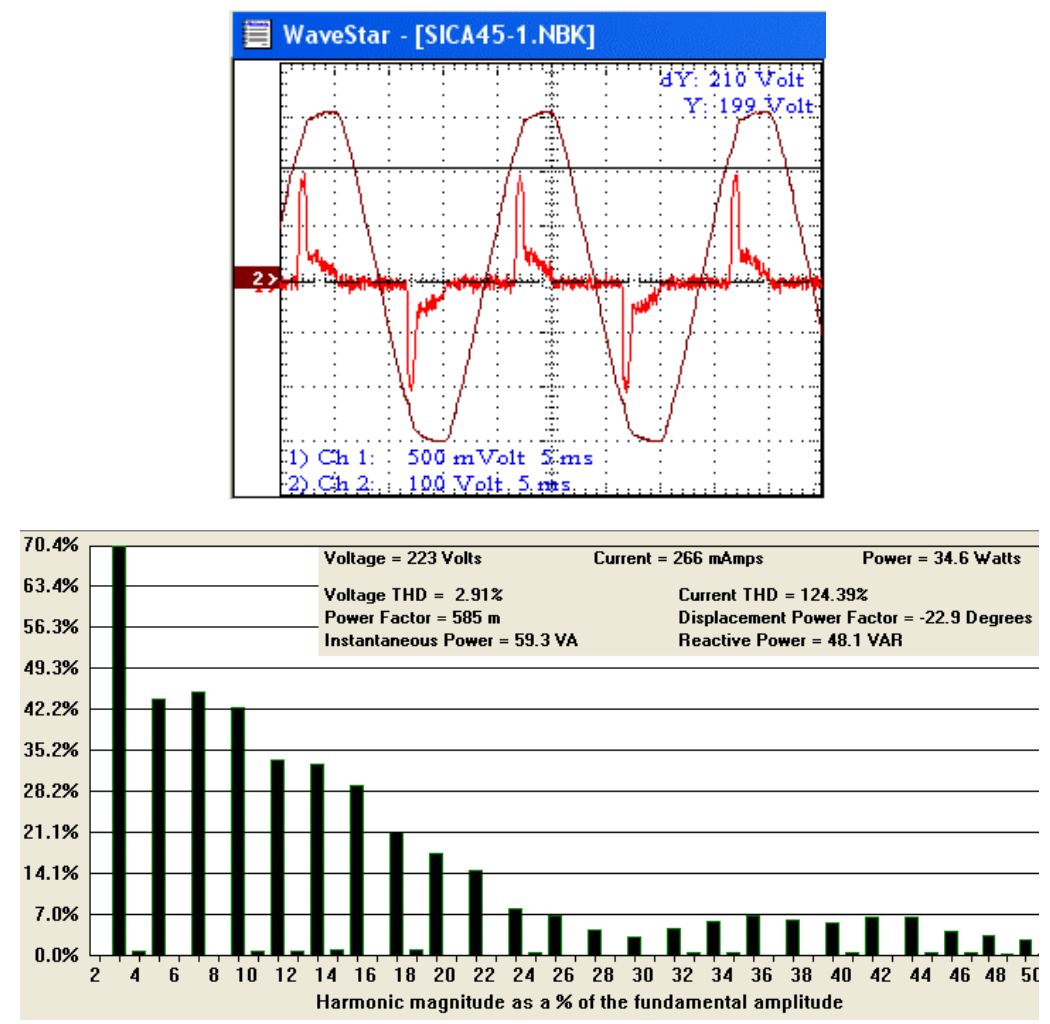

Figura 2. Formas de onda de tensión y corriente correspondiente a una lámpara de bajo consumo. Diagrama de contenido armónico para un THD de $124 \%$ con un coseno phi de $-0,92$ y un FP $=0,589$. Fuente: elaboración propia. 
Otro problema diferente es el de la modulación de alta frecuencia en la señal de corriente. Este fenómeno es sustancialmente diferente al del contenido armónico, ya que, a diferencia de este, el factor de potencia no se afecta en forma directa.

La modulación ocurre cuando en una carga electrónica basada en un circuito pulsador de alta frecuencia es conectada a la red de distribución sin el correspondiente filtro de radiofrecuencia. Dado que el circuito pulsador conecta y desconecta la carga a alta frecuencia para producir una reducción de la tensión de salida, es posible observar cómo la corriente se discontinúa. Como resultado la corriente de enterada se corresponderá con una señal modulante de $50 \mathrm{~Hz}$ y una portadora por encima de $40 \mathrm{KHz}$ [3].
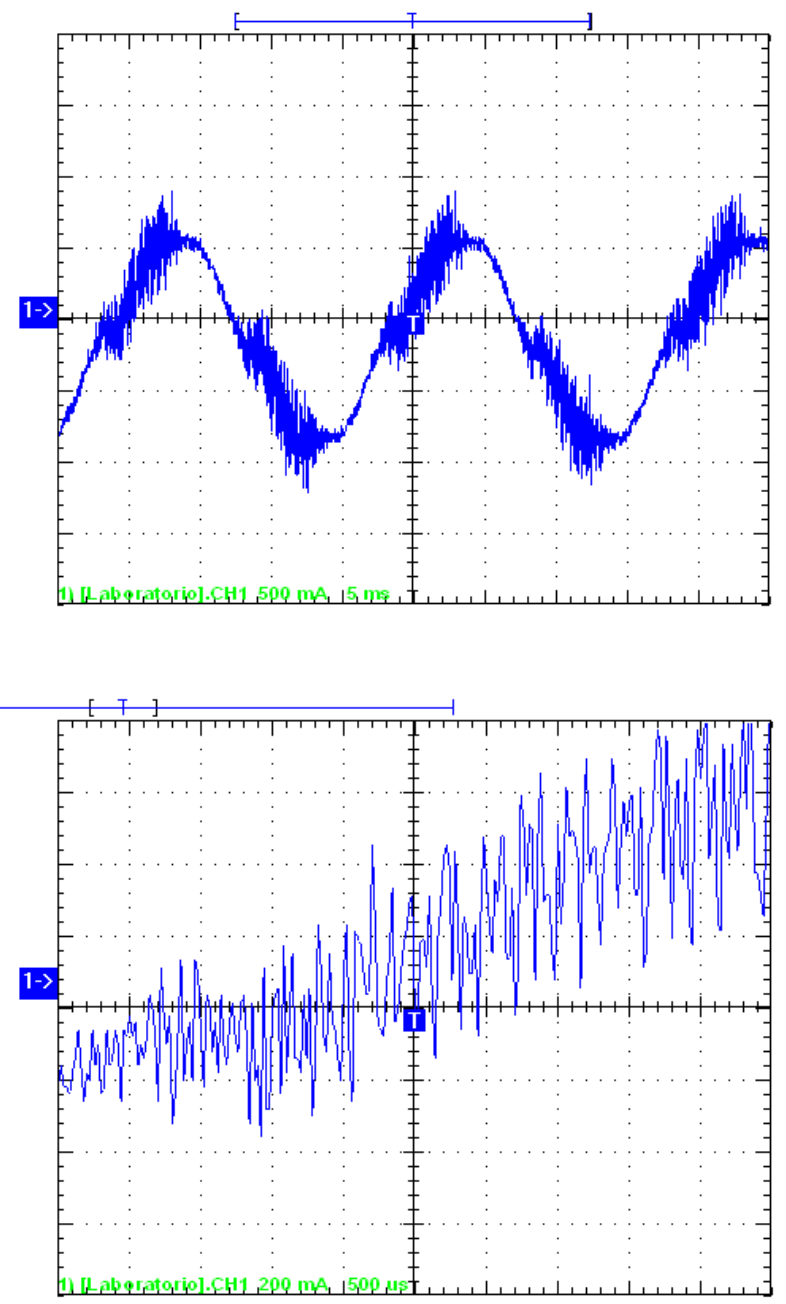

Figura 3. Oscilogramas que muestran la presencia de modulación de $\mathrm{HF}$ en corriente de línea de $50 \mathrm{~Hz}$, obtenida con un osciloscopio Tektronix TDS 1002B. Fuente: elaboración propia.
Este fenómeno es común en todas las tecnologías LED de baja potencia para iluminación, particularmente por la imposibilidad de insertar un filtro dentro del dispositivo.

\section{Metodología}

Para determinar el impacto del reemplazo de tubos fluorescentes con equipo auxiliar electromagnético por tubos de nueva tecnología LED se realizaron dos tipos de ensayos diferentes. Por una parte, se realizaron mediciones en los tableros de distribución del sis tema de iluminación. Por la otra, se realizaron mediciones de laboratorio. En ambos casos se utilizó como instrumento fundamental el osciloscopio Tektronix TDS 1002B con punta de corriente Fluke 510.

Con las mediciones en el tablero de distribución se busca determinar la forma de onda de corriente de fase cuando se encuentran en pleno funcionamiento el total de la carga de alimentación LED. El objetivo de esta medición es el de obtener en qué forma se presenta la señal de alta frecuencia en un sistema con un número importante de fuentes de señal modulante.

Con las mediciones de laboratorio se busca definir el comportamiento individual de cada uno de los tubos LED. Finalmente, el análisis comparativo de las mediciones realizadas sobre los tubos LED y sobre un tubo fluorescente conectado a un balasto electromagnético original busca documentar las mejoras en el consumo y particularmente la mejora en el factor de potencia.

\section{Resultados}

\subsection{Mediciones sobre el tablero de distribución}

Las mediciones que se realizaron sobre el tablero de distribución fueron únicamente de corriente. El osciloscopio antes mencionado no posee una adecuada aislación de la línea de alimentación, por lo que resulta inconveniente medir tensiones directamente. Se realizaron tomas de datos sobre cada una de las fases que alimentan el sistema de iluminación. Durante la medición el total de los tubos LED se encontraban en funcionamiento.

En la figura 4 pueden verse los oscilogramas correspondientes a dos fases diferentes medidas sobre el tablero de distribución. En estas puede observarse que la señal de corriente presenta distorsión asimétrica de cruce por cero. Además, no se observa una componente de muy alta frecuencia, sin embargo, se observa que existe algún grado de modulación sobre las señales de baja frecuencia. 
Evidentemente la distorsión armónica de la señal es mayor a la que presentaría la señal de tensión en la red. Dadas las características del tablero y del instrumento de medición no es posible definir el factor de potencia del sistema. Sin embargo, el instrumento de panel encargado de la medición del factor de desplazamiento indica un valor de 0,9. El valor de la corriente de fase 14,23 A. De este dato podría estimarse que en estas fases se encuentran conectados aproximadamente 200 tubos.

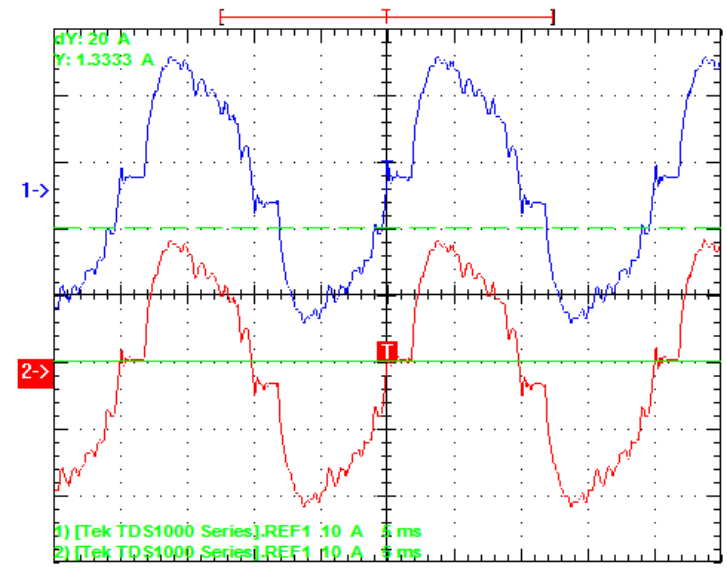

Figura 4. Formas de onda de corriente tomadas sobre dos fases diferentes del tablero de distribución. Fuente: elaboración propia.

\subsection{Mediciones de laboratorio}

\subsubsection{Tubos LED}

Uno de los ensayos de seguimiento que se realizan sobre la instalación de los dispositivos LED contempla la extracción de un ejemplar cada 6 meses de operación. Sobre estas muestras se realizan análisis eléctricos y de iluminancia. El objetivo es determinar las variaciones que presenten en sus parámetros en la medida que se produce el envejecimiento o se ven afectados por el ambiente.

Entre las mediciones que se realizaron sobre estos ejemplares se encuentran las de forma de onda de corriente, potencia activa y aparente, distorsión armónica total y determinación del valor de frecuencia de la componente de alta frecuencia. En la figura 5 se muestran las formas de onda de dos tubos en diferentes etapas de su vida. En ambos casos vemos que prevalece un gran contenido de señal de alta frecuencia con un período de pulsación de $10 \mu \mathrm{s}$. Además, es casi imposible de definir la distorsión de cruce por cero debido a esta componente. Si se combinan en un mismo oscilograma las señales de tensión y de corriente (en modo promedio de captura), vemos cómo ambas señales se encuentran prácticamente en fase y cómo la distorsión de cruce por cero se ubica en el centro del cruce de la tensión, pero en forma asimétrica (figura 6). Evidentemente el factor de desplazamiento es correcto, y, por lo tanto, no existe una componente reactiva de importancia.

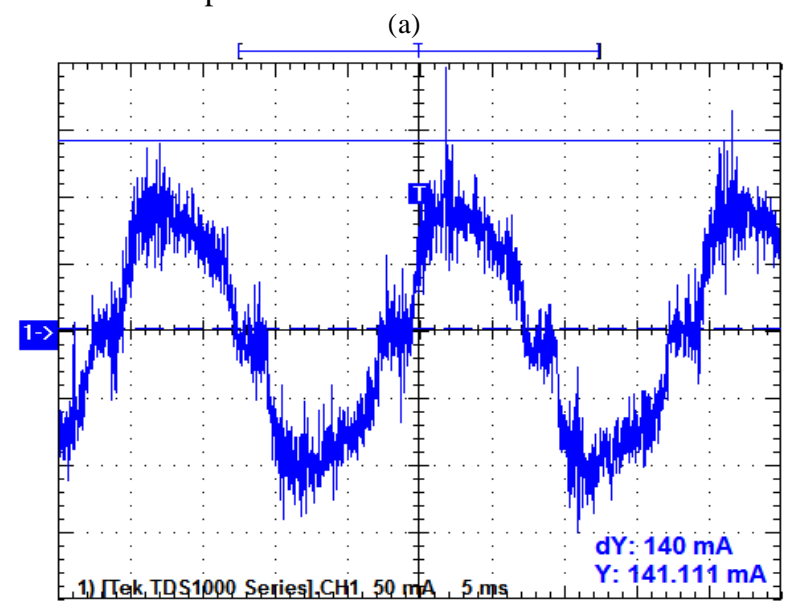

(b)

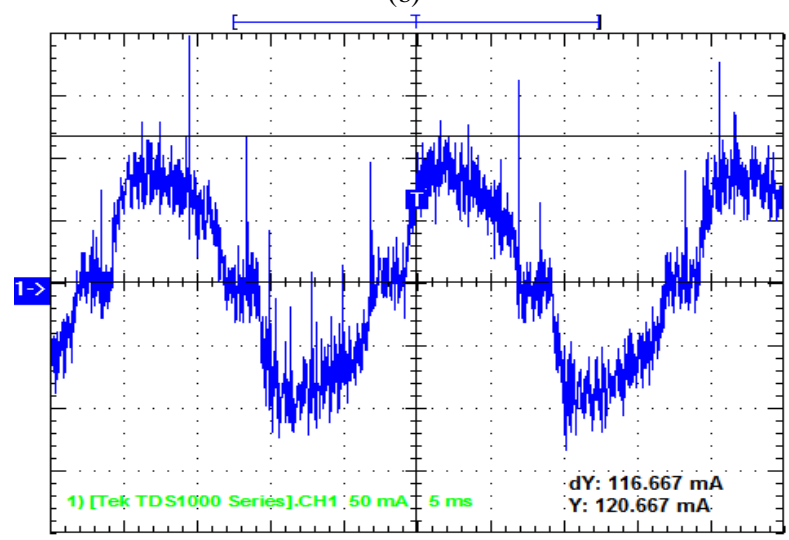

Figura 5. Formas de onda de corriente tomadas en bornes del tubo LED. (a) A los 3 meses de funcionamiento. (b) A los 9 meses. Fuente: elaboración propia.

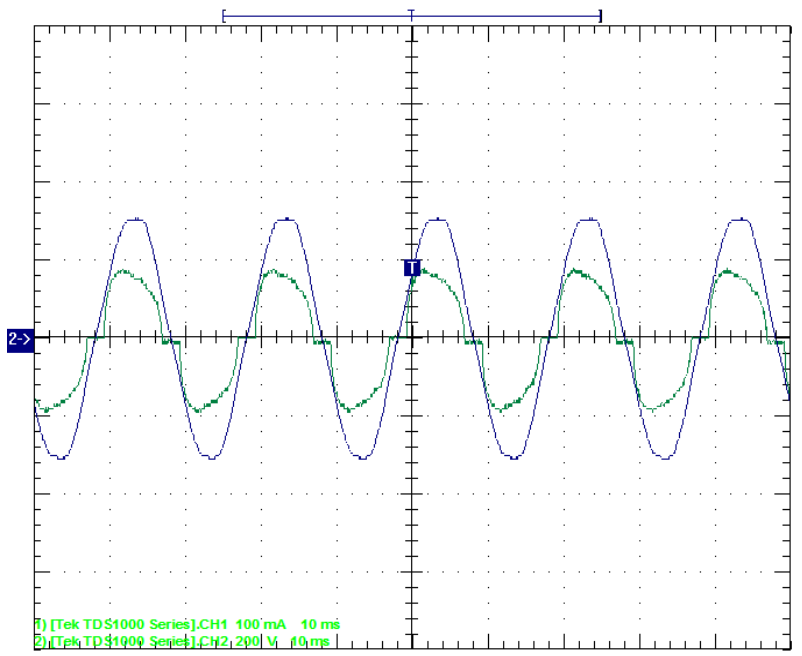

Figura 6. Formas de onda de corriente y tensión tomada en bornes del tubo LED en el laboratorio, en modo de captura promedio del osciloscopio Tektronix 1002B. Fuente: elaboración propia. 
De la determinación de los factores de caracterización de la potencia eléctrica consumida vemos que el factor de potencia declarado por el fabricante es incluso menor al factor de potencia medido en modo promedio por el osciloscopio. La potencia activa se ubica levemente por debajo del valor declarado por el fabricante y el factor de desplazamiento es de $7,24^{\circ}$ capacitivo.

Tabla 2. Captura de pantalla de análisis de potencia del software WaveStar de Tektronix. Tubo LED.

\begin{tabular}{|l|l|}
\hline Voltage $=222,59 \mathrm{v}$ & Current $=64,649 \mathrm{~mA}$ \\
\hline & \\
\hline Voltage THD $=3,146 \%$ & Current THD $=18,536 \%$ \\
\hline Power Factor $=972,07 \mathrm{~m}$ & $\begin{array}{l}\text { Dispacement Power Factor }=- \\
7,2486\end{array}$ \\
\hline $\begin{array}{l}\text { Apparent Power }=14,390 \\
\text { VA }\end{array}$ & Reactive Power= 3,3771 VAR \\
\hline True Power $=13,988 \mathrm{~W}$ & \\
\hline
\end{tabular}

Fuente: elaboración propia.

La distorsión armónica total se ubica en el 18,53 \% para la corriente y en el 3,14 \% para la tensión. Estos valores se encuentran dentro de todas las normativas internacionales que estandarizan cargas de iluminación de potencias inferiores a $70 \mathrm{~W}$.

Si se repite el mismo ensayo, pero con uno de los balastos originales, de fabricación alemana, del año 1.968, es posible realizar un análisis comparativo de ambas tecnologías.

\subsubsection{Balasto electromagnético}

Si se observan las formas de onda del balasto electromagnético original, es posible identificar una forma de onda de corriente con una distorsión armónica moderada, pero con un desplazamiento en retardo notable.

Los valores de corriente consumida alcanzan los 413,44 mA. Valores, superiores en varios órdenes a los registrados en las lámparas de LED. Sin embargo, valores esperados en un balasto electromagnético con una lámpara fluorescente sin capacitor de compensación, pero tal vez inesperados en un dispositivo de más de 45 años de antigüedad y en un funcionamiento continuo.

Las formas de onda se muestran en la figura 7 mientras que en tabla 3 se detallan los valores del análisis de potencia.

Donde nuevamente esta última figura corresponde a una captura de pantalla obtenida del software del osciloscopio.
Del análisis de potencia resulta claro que el balasto electromagnético presenta un factor de desplazamiento elevado: $55,14^{\circ}$ inductivo .Una distorsión armónica del $12 \%$ con igual valor de THD de tensión registrado en el ensayo anterior. Como consecuencia, la componente reactiva alcanza un valor elevado 75,52 VAR y el factor de potencia resulta bajo 0,56 .

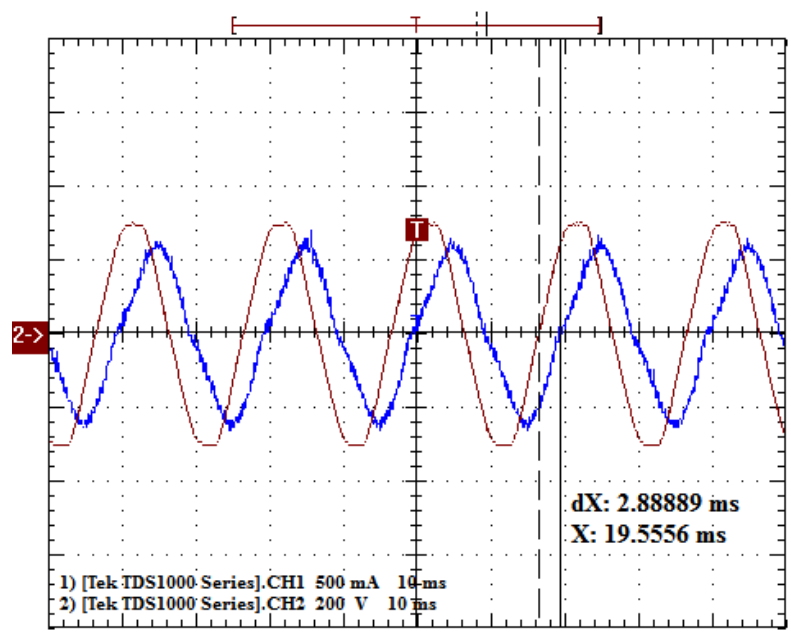

Figura 7. Formas de onda de tensión y corriente para un balasto electromagnético obtenido de la instalación original (año 1968). Fuente: elaboración propia.

Tabla 3. Captura de pantalla de análisis de potencia del software WaveStar de Tektronix. Balasto electromagnético.

\begin{tabular}{|l|l|}
\hline Voltage $=220,66 \mathrm{v}$ & Current $=413,44 \mathrm{~mA}$ \\
\hline & \\
\hline Voltage THD=3,176\% & Current THD=12,011\% \\
\hline Power Factor=561,06 $\mathrm{m}$ & $\begin{array}{l}\text { Dispacement Power Factor= } \\
55.145\end{array}$ \\
\hline $\begin{array}{l}\text { Apparent Power= 91.231 } \\
\text { VA }\end{array}$ & Reactive Power= 75.519 VAR \\
\hline True Power=51.186 W & \\
\hline
\end{tabular}

Fuente: elaboración propia.

\subsubsection{Análisis comparativo}

Si se realiza un análisis comparativo del contenido armónico para cada uno de los dispositivos, tal como se muestra en la figura 8 y en la figura 9 , vemos que existen diferencias sustanciales en la distribución espectral. En primer lugar, vemos que el contenido armónico del balas to electromagnético se concentra fundamentalmente en la tercera armónica, mientras que en el caso de la lámpara LED se presenta un espectro con mayor cantidad de componentes con valores significativos. 


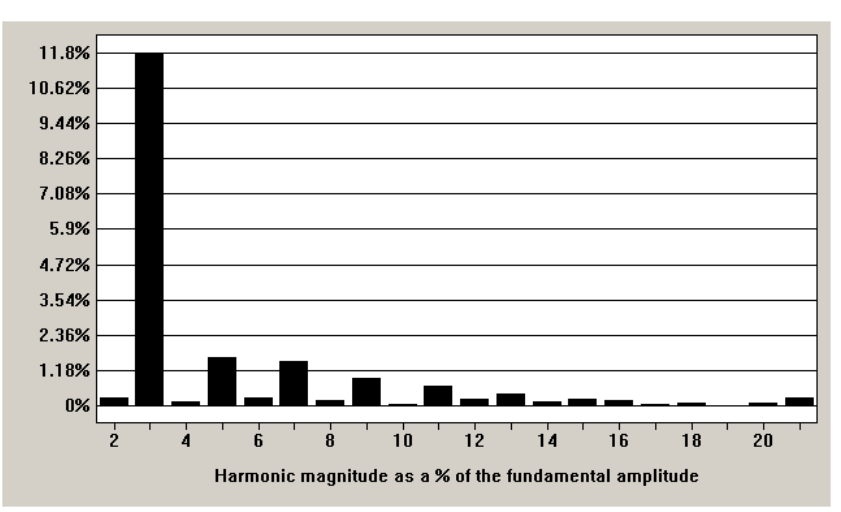

\begin{tabular}{|l|r|r|r|r|}
\hline & Frequency & $\begin{array}{c}\text { Current } \\
\text { RMS }\end{array}$ & $\begin{array}{c}\text { Current } \\
\text { \% of Fund. }\end{array}$ & $\begin{array}{c}\text { Current } \\
\text { Phase }\end{array}$ \\
\hline Fundamental & $50,000 \mathrm{~Hz}$ & $406,52 \mathrm{~mA}$ & 100 \% & 0,0000 \\
\hline Harmonic 2 & $100,000 \mathrm{~Hz}$ & $1,2007 \mathrm{~mA}$ & $0,295 \%$ & $-57,096$ \\
\hline Harmonic 3 & $150,00 \mathrm{~Hz}$ & $47,688 \mathrm{~mA}$ & $11,731 \%$ & 85,865 \\
\hline Harmonic 4 & $200,00 \mathrm{~Hz}$ & $533,20 \mathrm{uA}$ & 0,131 \% & -47.227 \\
\hline Harmonic 5 & $250,00 \mathrm{~Hz}$ & $6,6219 \mathrm{~mA}$ & $1,629 \%$ & 127,53 \\
\hline Harmonic 6 & $300,00 \mathrm{~Hz}$ & $1,0583 \mathrm{~mA}$ & $0,260 \%$ & $-37,200$ \\
\hline Harmonic 7 & $350,00 \mathrm{~Hz}$ & $6,0880 \mathrm{~mA}$ & $1,498 \%$ & 75,073 \\
\hline Harmonic 8 & $400,00 \mathrm{~Hz}$ & $831,88 \mathrm{uA}$ & $0,205 \%$ & $-105,86$ \\
\hline Harmonic 9 & $450,00 \mathrm{~Hz}$ & $3,7579 \mathrm{~mA}$ & $0,924 \%$ & 36,661 \\
\hline Harmonic 10 & $500,00 \mathrm{~Hz}$ & $278,08 \mathrm{uA}$ & $0,068 \%$ & 97,588 \\
\hline Harmonic 11 & $550,00 \mathrm{~Hz}$ & $2,6682 \mathrm{~mA}$ & 0,656 \% & 39,254 \\
\hline Harmonic 12 & $600,00 \mathrm{~Hz}$ & $915,74 \mathrm{uA}$ & $0,225 \%$ & 156,70 \\
\hline Harmonic 13 & $650,00 \mathrm{~Hz}$ & $1,7598 \mathrm{~mA}$ & $0,433 \%$ & $-20,899$ \\
\hline Harmonic 14 & $700,00 \mathrm{~Hz}$ & $689,16 \mathrm{uA}$ & 0,170 \% & 30,805 \\
\hline Harmonic 15 & $750,00 \mathrm{~Hz}$ & $1,0548 \mathrm{~mA}$ & 0,259 \% & 30,745 \\
\hline Harmonic 16 & $800,00 \mathrm{~Hz}$ & $805,81 \mathrm{uA}$ & $0,198 \%$ & $-40,699$ \\
\hline
\end{tabular}

Figura 8. Tablas de análisis espectral y gráficos obtenidos del software WaveStar. Balasto electromagnético. Fuente: elaboración propia.

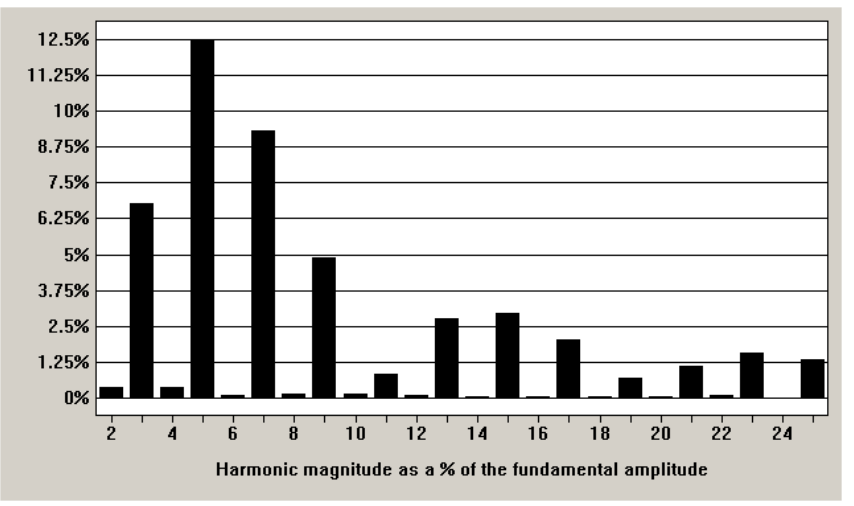

\begin{tabular}{|c|c|c|c|c|}
\hline & Frequency & $\begin{array}{c}\text { Current } \\
\text { RMS }\end{array}$ & $\begin{array}{c}\text { Current } \\
\% \text { of Fund. }\end{array}$ & $\begin{array}{l}\text { Current } \\
\text { Phase }\end{array}$ \\
\hline ndamental &, 100114 & $63,318 \mathrm{~mA}$ & $100 \%$ & \\
\hline armonic 2 & & $254,47 \mathrm{uA}$ & $402 \%$ & 51,29 \\
\hline & & & & \\
\hline & & & & \\
\hline$m$ & & 7,87 & & \\
\hline & & 66,8 & & $-5,578$ \\
\hline & & & & 38,7 \\
\hline 8 & 400 & 98, & & \\
\hline nic 9 & 450 & $3,1070 \mathrm{~mA}$ & $907 \%$ & 51,4 \\
\hline & & & & \\
\hline 11 & & 54 & & 166 \\
\hline Harm & 601, & $76,068 \mathrm{uA}$ & $20 \%$ & -133 \\
\hline 13 & & 1,77 & & 5 \\
\hline & & & & \\
\hline & & & & \\
\hline armonic 16 & 801 , & $31,183 \mathrm{uA}$ &, 04 & 15, \\
\hline
\end{tabular}

Figura 9. Tablas de análisis espectral y gráficos obtenidos del software WaveStar. Tubo LED. Fuente: elaboración propia.

En el caso del tubo LED la armónica de mayor orden no es la tercera, sino la quinta, seguida por la séptima. En general, en ninguna de las señales se presentan armónicos pares o contenido de DC.

Es evidente que la señal de alta frecuencia no es tenida en cuenta en el análisis espectral, dado que el osciloscopio y el software trabajan sobre una señal promediada durante el proceso de captura.

\section{Conclusiones}

Una vez realizadas las mediciones de campo y las de laboratorio, se puede concluir que el reemplazo de tubos fluorescentes por nuevos tubos de tecnología LED resulta sumamente conveniente. La sola comparación de los valores de potencia activa reactiva y aparente entre ambas tecnologías muestra una notable mejoría en la incorporación de tecnología de estado sólido. La reducción de potencia activa es notable, pasa de $52 \mathrm{~W}$ a 
14 W. Sin embargo, resulta todavía mejor la reducción de la potencia aparente.

Si se realiza un análisis de la mejora en el factor de potencia, vemos que esta se produce en la instalación de tubos LED a partir de una reducción notable en el factor de desplazamiento: $7,25^{\circ}$ capacitivos respecto de los $55,14^{\circ}$ inductivos. Además, al tratarse de una pequeña carga capacitiva en conjunto tiende a compensar cargas inductivas presentes en el sistema eléctrico, por ejemplo, los motores de ventilación, a partir de la incorporación de 14 KVAR

Si se analiza el factor armónico, vemos que en el caso de los balastos electromagnéticos los parámetros son favorables, principalmente por el hecho de concentrar la mayor parte de la distorsión en el tercer armónico. Componente que resultaría nulo en presencia de un sistema trifásico equilibrado.

En su conjunto el factor de potencia y el consumo del sistema de iluminación presentan ventajas notables al aplicar este nuevo tipo de tecnologías.

Quedan por definir en un anális is más profundo los reales efectos de las señales de alta frecuencia generadas por cada uno de los pulsadores que gobiernan los LED. En un principio, y con las herramientas de medición dis ponibles, es posible apreciar que, de alguna forma la interferencia de múltiples señales de HF sobre la línea de alimentación tiende a atenuar notablemente el contenido de estas señales, y se reduce el impacto sobre el sistema eléctrico. Sin embargo, estas mediciones no son determinantes, y, por lo tanto, es necesario profundizar en este estudio, y aplicar, además, mediciones de campo electromagnético de alta frecuencia en el viaducto.

\section{Referencias}

[1] D. M. N. Balducci, F. M. Vincitorio, J. L. Frund, L. D. Rispoli, C. D. Mache. Active power measurement in the presence of harmonic currents. IEEE Argencon - 2012 Córdoba. Argentina.

[2] M. H. Rashid, "Power Electronics: Circuits, Device and Applications," 3rd ed. New Jersey, E.E.U.U.: Pearson Education Inc, 2003.

[3] J. L. Frund, F. M. Vincitorio, D. M. N. Balducci, C.E. Freyre, A. Chort, J Favre. High Frequencies in AC Distribution Lines: a New Technical Challenge. VII International Symposium on Power Quality. SICEL 2013. Medellin, Colombia.
[4] R. I. Sasaki. (1994, Sep.). The Impact Of Electronic Ballast Compact Fluorescent Lighting On Power Distribution Systems. Purdue University. West Lafayette, Indiana. [Online]. Available: http://docs .lib.purdue.edu/ecetr/197/

[5] W. C. Cortés Jiménez et al., "Identificación y análisis de componentes de frecuencia entre $10 \mathrm{KHz}$ y $200 \mathrm{KHz}$ causadas por bombillas compactas fluorescentes," in XI Congreso Iberoamericano de Iluminación Luxamérica., Cartagena, Colombia, Oct. 9-12, 2012, pp.60

[6] ELI Technical Documents for Certification. First edition 2006-03-01. ELI Voluntary Technical Specification for Self-Ballasted Compact Fluorescent Lamps (CFLs). ELI Quality Certification Institute.

[7] Z. Radakorvic, F Topalis, M. Kostic. The voltage distortion in low - voltage networks caused by fluorescent lamps with electronic gear. Electronic Power System Research 73 (2005) 129-136. Elsevier. 